\title{
Precondition for discretized fractional boundary value problem
}

\author{
I. K. Youssef ${ }^{1,}$, , A. M. Shukur ${ }^{2}$ \\ ${ }^{1}$ Department of Mathematics, Ain Shams University, Cairo, Egypt \\ ${ }^{2}$ Department of Applied Mathematics, University of Technology, Baghdad, Iraq
}

\section{Email address:}

kaoud22@hotmail.com(I. K. Youssef), ahmhshald@yahoo.com(A. M. Shukur)

\section{To cite this article:}

I. K. Youssef, A. M. Shukur. Precondition for Discretized Fractional Boundary Value Problem. Pure and Applied Mathematics Journal. Vol. 3, No. 1, 2014, pp. 1-6.doi: 10.11648/j.pamj.20140301.11

\begin{abstract}
A precondition that uses the special structure of the algebraic system arising from the discretization of a fractional partial differential equation on the red black ordering grid is introduced. Comparison with the numerical solution of the classical Poisson's equationis considered. A graphical representation for the precondition is illustrated. The performance of our treatment is calculated for different values of the fractional order. The results of the implementation of the SOR and the KSOR with the help of MATLAB are given in compact tables. The value of the relaxation parameter is chosen on the bases of the graphical representation of the behavior of the spectral radius of the iteration matrices. Also, the natural ordering grid is considered.
\end{abstract}

Keywords: Fractional Boundary Value Problem, SOR, KSOR and Precondition

\section{Introduction}

Differential and integral equations are used in modeling physical phenomena. In recent years a considerable amount of progress has been made in using fractional order partial differential equations (FPDE) in modeling different fields of science. FPDE can be seen as a generalization of the classical partial differential equations (PDE) in the sense that it takes into account the memory and hereditary properties of the physical phenomena. As it was in the classical PDE there is no general method that can be used in solving FPDE. Numerical solution of FPDE has received great progress in the recent years. There is no doubt that the work of Young introduced in his Ph.D. thesis in 1950 as well as in his famous book in 1971 represents a major shift in the developments of the numerical treatment of structured large linear systems that arise from the discretization of PDE. Young introduced the successive over relaxation method SOR during his numerical treatment of Poisson's equation $[1$, 2].Young,also considered the methods of labeling the grid points and their effect on the structure of the resultant linear algebraic system. Youssef in 2012 introduced a new variant (KSOR) of the SOR method [3]. Precondition is a technique used in the recent years to adopt the convergence of iterative methods. Our aim is to introduce a precondition suitable to discretize FPDE and to compare its performance when applied to both SOR and KSOR methods. The Poisson's equation is:

$$
-\left(\frac{\partial^{2}}{\partial x^{2}}+\frac{\partial^{2}}{\partial y^{2}}\right) \mathrm{u}(\mathrm{x}, \mathrm{y})=\mathrm{f}(\mathrm{x}, \mathrm{y}),(x, y) \in D
$$

Where, $\mathrm{D}=\{(\mathrm{x}, \mathrm{y}):(\mathrm{x}, \mathrm{y}) \in[0, \mathrm{~L}] \times[0, \mathrm{~L}]$

Subject to the Dirichlet-boundary conditions:

$$
\mathrm{u}(0, \mathrm{y})=\mathrm{u}(\mathrm{x}, 0)=\mathrm{u}(\mathrm{L}, \mathrm{y})=0 ; \text { and } \mathrm{u}(\mathrm{x}, \mathrm{L})=\mathrm{g}(\mathrm{x}) .(2)
$$

Also[4]considered the fractional Poisson's equation, in the form:

$$
-\left(\frac{\partial^{\alpha}}{\partial \mathrm{x}^{\alpha}}+\frac{\partial^{\alpha}}{\partial y^{\alpha}}\right) \mathrm{u}(\mathrm{x}, \mathrm{y})=\mathrm{f}(\mathrm{x}, \mathrm{y}), \text { where } 1<\alpha \leq 2,
$$

Subject to the same boundary conditions in (2) and he used the finite difference method to approximate the fractional derivatives

We consider the corresponding fractional order Elliptic FPDE by the form:

$$
-\left(\frac{\partial^{\alpha}}{\partial x^{\alpha}}+\frac{\partial^{\beta}}{\partial y^{\beta}}\right) u(x, y)=f(x, y) ;(1<(\alpha \text { and } \beta) \leq 2)(4)
$$

Note1: equation (4) can be seen as a generalization to both equation (1) and equation (3). 


\section{Finite Difference Approximations}

It is well known that the five point's finite difference approximation for equation (1) can be written in the form

$$
4 U_{i, j}-U_{i-1, j}-U_{i+1, j}-U_{i, j-1}-U_{i, j+1}=h^{2} f_{i j}(5)
$$

In order to obtain the finite difference approximation of the fractional order equation (3), we use the treatment introduced in [4] for approximate Caputo's fractional derivative of order $\alpha,(1<\alpha \leq 2)$ in the form:

$$
\begin{aligned}
{ }^{C} D_{x_{i}^{+}}^{\alpha} u\left(x_{i}, y_{j}\right) \approx \frac{U_{i+1, j}-2 U_{i, j}+U_{i-1, j}}{(3-\alpha) h^{\alpha}} & \text { and } \\
& { }^{C} D_{y_{j}^{+}}^{\alpha} u\left(x_{i}, y_{j}\right) \approx \frac{U_{i, j+1}-2 U_{i, j}+U_{i, j-1}}{(3-\alpha) h^{\alpha}}(6 \mathrm{a})
\end{aligned}
$$

It is well known that the central finite difference approximation of the second order derivative is given by:

$$
\begin{aligned}
& \frac{\partial^{2} u\left(x_{i}, y_{j}\right)}{\partial x^{2}} \approx \frac{U_{i+1, j}-2 U_{i, j}+U_{i-1, j}}{h^{2}}-R_{h}(x), \\
& \text { Where, }
\end{aligned}
$$

$$
\left|R_{h}(x)\right| \leq \sup _{x}\left|u^{(4)}(x, y)\right| h^{2} / 12,
$$

where $R_{h}$ is the truncation error.

The corresponding finite difference approximation of the Caputo's fractional order derivative of order $\alpha$ is:

$$
\frac{U_{i+1, j}-2 U_{i, j}+U_{i-1, j}}{(3-\alpha) h^{\alpha}} \approx{ }^{C} D_{x_{i}^{+}}^{\alpha} u\left(x_{i}, y_{j}\right)+\frac{h^{4-\alpha} \max \left|u^{(4)}\left(x_{i}\right)\right|}{12(3-\alpha)}
$$

Similarly,

$$
\frac{U_{i, j+1}-2 U_{i, j}+U_{i, j-1}}{(3-\alpha) h^{\alpha}} \approx{ }^{C} D_{y_{j}^{+}}^{\alpha} u\left(x_{i}, y_{j}\right)+\frac{h^{4-\alpha} \max \left|u^{(4)}\left(y_{j}\right)\right|}{12(3-\alpha)}(6 \mathrm{~d})
$$

Accordingly, the difference approximation for eqn. (3) can be written in the form:

$$
\frac{U_{i+1, j}-2 U_{i, j}+U_{i-1, j}}{(3-\alpha) h^{\alpha}}+\frac{U_{i, j+1}-2 U_{i, j}+U_{i, j-1}}{(3-\alpha) h^{\alpha}}=-f_{i, j},
$$

which can be rearranged as in the classical form (5) as:

$$
4 U_{i, j}-U_{i-1, j}-U_{i+1, j}-U_{i, j-1}-U_{i, j+1}=(3-\alpha) h^{\alpha} f_{i j}(7)
$$

Also there is another two treatments in approximating the fractional derivatives inequation (4),

The first treatment is the direct discretization methodin this method the integral in Caputo's formula is replaced by a finite sum of integrals at the discretization points, and approximatethe second orderderivative by using the standard finite difference formula, then the finite difference formula of Caputo's fractional derivative takes the form:

$$
\begin{array}{r}
{ }_{0}^{C} D_{x}^{\alpha} u(x, y) \cong \frac{1}{\Gamma(2-\alpha)} \sum_{k=0}^{i-1} \int_{x_{k}}^{x_{k+1}} \frac{\partial^{2} u(s, y)}{\partial s^{2}}(x-s)^{2-\alpha-1} d s \\
{ }_{0}^{C} D_{x}^{\alpha} u_{i, j} \cong \frac{1}{h^{\alpha} \Gamma(3-\alpha)} \sum_{k=0}^{i-1} b_{k}^{\alpha}\left(U_{i-k+1, j}-2 U_{i-k, j}+\right. \\
\left.+U_{i-k-1, j}\right)
\end{array}
$$

Where, $b_{k}^{\alpha}=\left[(k+1)^{2-\alpha}-k^{2-\alpha}\right]$, let $b_{s}^{*}=\frac{-1}{h^{\beta} \Gamma(3-\beta)} b_{s}^{\beta} ; b_{k}=\frac{-1}{h^{\alpha} \Gamma(3-\alpha)} b_{k}^{\alpha}$

Then, the finite difference scheme for eqn(4) take the form:

$$
\begin{array}{r}
\sum_{k=0}^{i-1} b_{k}\left(U_{i-k+1}^{j}-2 U_{i-k}^{j}+U_{i-k-1}^{j}\right)+ \\
+\sum_{s=0}^{j-1} b_{s}^{*}\left(U_{i}^{j-s+1}-2 U_{i}^{j-s}+U_{i}^{j-s-1}\right)=f_{i}^{j}
\end{array}
$$

It is clear that when $\alpha=\beta$, one can easily see that:

$$
b_{s}^{*}=b_{k}=\frac{-1}{h^{\alpha} \Gamma(3-\alpha)} b^{\alpha} \text {. }
$$

Also, the system in (7) can be obtained from (9) by taking the first term in the sum with $b_{0}=1$ for all $1<\alpha \leq 2$.

The second treatmentfor the fractional derivatives [5-7],

$$
\begin{gathered}
{ }_{0}^{R-L} D_{x}^{\alpha} u\left(x_{i}, y_{j}\right)={ }_{0}^{R-L} D_{x}^{\alpha} u\left(x_{i}, y_{j}\right) \\
=\lim _{\Delta x \rightarrow 0} \frac{1}{(\Delta x)^{\alpha}} \sum_{k=0}^{N} w_{k}^{\alpha} U_{N-k, j} ; w_{k}^{\alpha} \\
=\frac{\Gamma(k-\alpha)}{\Gamma(k+1) \Gamma(-\alpha)}
\end{gathered}
$$

$$
\begin{aligned}
{ }_{0}^{C} D_{x}^{\alpha} u(x, y) & \\
= & { }_{0}^{R-L} D_{x}^{\alpha} u(x, y)-\sum_{k=0}^{n-1} \frac{x^{k-\alpha}}{\Gamma(k+1-\alpha)} \frac{\left.\partial^{k} u(x, y)\right|_{x=0}}{\partial x^{k}} \\
& \quad{ }_{0}^{G-L} D_{x}^{\alpha} u\left(x_{i}, y_{j}\right)=\frac{1}{h^{\alpha}} \sum_{k=0}^{i+1} g_{k}^{\alpha} U_{i-k+1, j}+O(h),
\end{aligned}
$$

Depending on the use of Grunwald-Letnikov (G-L) [5]).

Accordingly, the finite difference approximation to eqn(4), take the form:

$$
\sum_{k=0}^{i+1} g_{k}^{\alpha} U_{i-k+1}^{j}+\sum_{s=0}^{j+1} g_{s}^{\beta} U_{i}^{j-s+1}=f_{i}^{j}(10)
$$

Where $f_{i}^{j}=f\left(x_{i}, y_{j}\right) ; U_{i, j}=U\left(x_{i}, y_{j}\right)$,

$$
\begin{gathered}
\left(x_{i}, y_{j}\right) \in D, x_{i}=i h ; y_{j}=j h ; h=\frac{L}{N} \\
z_{0}^{\gamma}=1 ; z_{1}^{\gamma}=-\gamma ; z_{k}^{\gamma}=\frac{\gamma(\gamma-1) \cdots(\gamma-k+1)}{k !} ; k>1 ; \\
g_{k}^{\alpha}=-r_{1} z_{k}^{\alpha} ; g_{s}^{\beta}=-r_{2} z_{k}^{\beta} ; r_{1}=\left(\frac{1}{h^{\alpha}}\right), r_{2}=\left(\frac{1}{h^{\beta}}\right)
\end{gathered}
$$

Inthe finite difference treatment the PDE or the FPDE are replaced by an Algebraicsystem of equations which can be written in the form

$$
\mathrm{AU}=\mathrm{f}
$$

where $A$ is $(\mathrm{N}-1)^{2} \times(\mathrm{N}-1)^{2}$ coefficients matrix, $\mathrm{U}$ and $\mathrm{f}$ are two $(\mathrm{N}-1)^{2} \times 1$ matrices, where $U=\left[U_{j}^{1}, U_{j}^{2}, \cdots, U_{j}^{N-1}\right]^{T}$ and $f=\left[f_{j}^{1}, f_{j}^{2}, \cdots, f_{j}^{N-1}\right]^{T}$; $j=1, \ldots, N-1$.

Usually the system in (12) is very large and the matrix of coefficientsA has special structure. 


\section{Coefficients Matrix of the Linear Algebraic System}

The structure of the matrix of coefficients depends on the method of discretizing, the domain and the labeling of the grid points

$$
\begin{aligned}
& \text { Let } f_{i}^{j}=f\left(x_{i}, y_{j}\right) ; U_{i, j}=U\left(x_{i}, y_{j}\right), \\
& \left(x_{i}, y_{j}\right) \in D, x_{i}=i h ; y_{j}=j h ; h=\frac{L}{N^{\prime}}
\end{aligned}
$$

Labeling of grid points [8]: in the finite difference treatment of classical PDE there are different methods of labeling the grid points, two of them are the Lexicographic ordering figures1 a and the Chequer-board ordering (Red-Black ordering) figures 1.b.

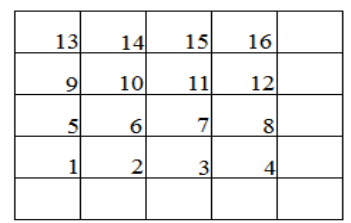

Figure1 a.Lexicographical ordering.

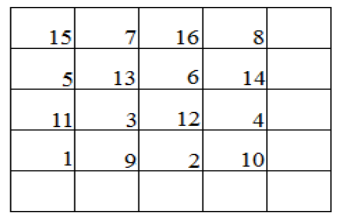

Figure1b.Chequer-board ordering

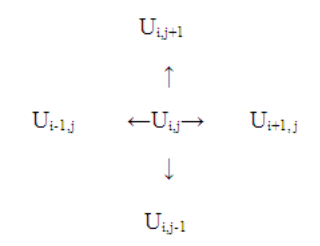

Figure 1c.

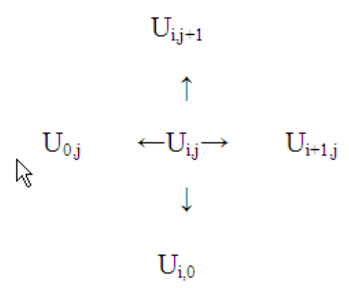

Figure 1d.

Figure 1c illustrate the linking between the function values at the grid points for classical (integer) case and figure $1 \mathrm{~d}$ illustrate the linking between the function values at the grid points for fractional order case, it is clear that the memory and hereditary properties of fractional derivatives can be noticed from figure $1 \mathrm{~d}$.
It is well known that the coefficient matrix for the algebraic system corresponding to equation (5) or equation (7) with the labeling of grid points in figures( $1 \mathrm{a}$ and $1 \mathrm{~b}$ )can be written in the block form (figures 2 and 3 ).

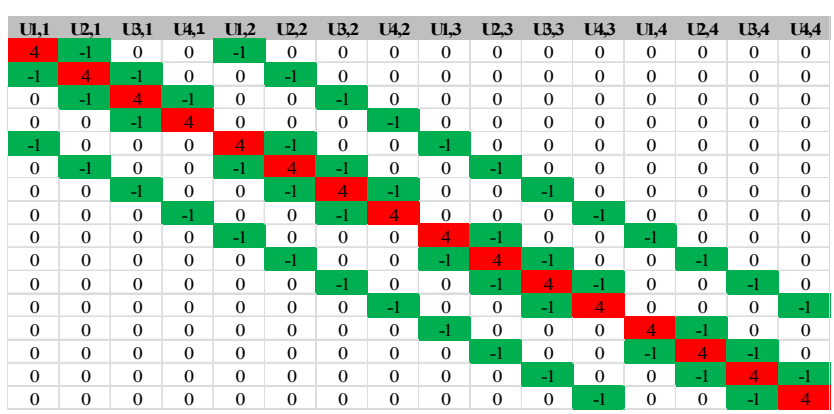

Figure2.Lexicographical ordering for formula (5 and 7) at $N=5$.

This can be described as the following block structure.

$$
A=\left\{\begin{array}{c}
\text { Bifk } k=l \\
-1 \text { Iifl }=k \mp 1, B \\
0 \quad \text { otherwise }
\end{array}=\left\{\begin{array}{c}
4 \text { if } j=i \\
-1 \text { if } j=i \mp 1, \\
0 \quad \text { otherwise }
\end{array}\right.\right.
$$

WhereI denotes the identity matrix of order 4 and $\mathrm{B}$ is a block sub-matrix of order 4 .

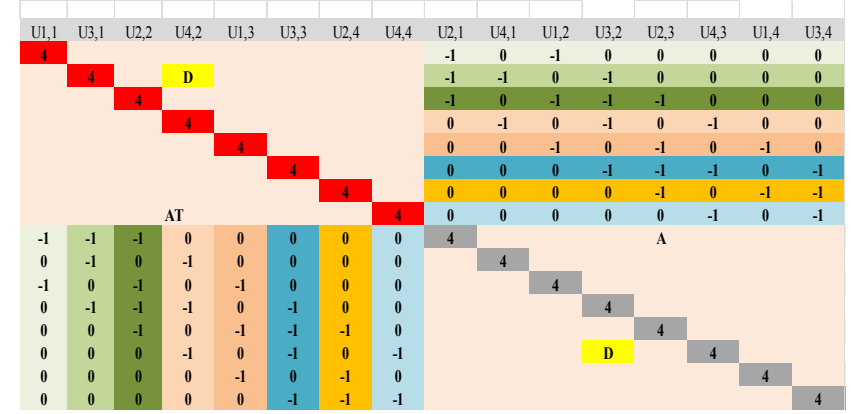

Figure3. Chequer-board ordering for formula (5 and 7) at $N=5$.

It is clear that the coefficients-matrix for the fractional order case equation (7), has the same structure as in equation (13) and the difference will be in the free column $\mathrm{f}$ in the right side of system (12).

Itshould be noticed that this representation does not reflect the memory and hereditary properties of fractional derivatives.

The matrix representation of the finite difference scheme in equation (9) or equation (10) with respect to the labeling of grid points in figures (1a and $1 \mathrm{~b}$ ) can be written in the following forms:

The coefficients-matrix of the system in equation (10) is:

$$
\begin{gathered}
A=\left\{\begin{array}{cc}
B 1 & \text { for } k=l \\
g_{c+1}^{*} I & \text { for } k-l=c=1,2 \ldots N-1 \\
g_{0}^{*} & \text { for } j=i-1 \\
0 & \text { otherwise }
\end{array}\right. \\
B 1=\left\{\begin{array}{cc}
g_{1}+g_{1}^{*} \quad \text { for } j=i \\
g_{t+1} & \text { for } i-j=t=1,2, \ldots N-1 \\
g_{0} & \text { for } j=i-1 \\
0 & \text { otherwise }
\end{array}\right.
\end{gathered}
$$




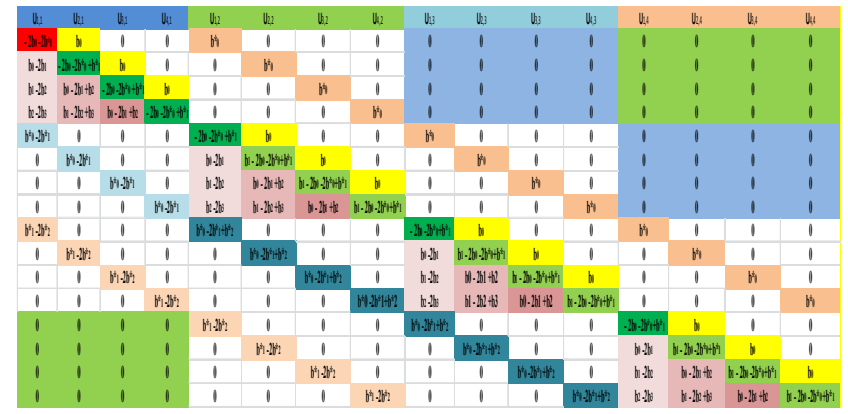

Figure4.Lexicographical ordering, formula (9), $1 \leq \alpha ; \beta \leq 2$.

The special case of system in equation (9) for $\alpha=\beta$, and $g_{k}^{\alpha}=g_{s}^{\beta}$, then the coefficients-matrix take the form:

$$
\begin{aligned}
\mathrm{B} 1 & =\left[\begin{array}{ccccccc}
2 g_{1} & g_{0} & 0 & \ldots & 0 & 0 & 0 \\
g_{2} & 2 g_{1} & g_{0} & \ldots & 0 & 0 & 0 \\
g_{3} & g_{2} & 2 g_{1} & & 0 & 0 & 0 \\
\vdots & \vdots & \vdots & \ddots & g_{0} & \vdots & \vdots \\
g_{N-3} & g_{N-4} & g_{N-5} & \ddots & 2 g_{1} & g_{0} & 0 \\
g_{N-2} & g_{N-3} & g_{N-4} & \ldots & g_{2} & 2 g_{1} & g_{0} \\
g_{N-1} & g_{N-2} & g_{N-3} & & g_{3} & g_{2} & 2 g_{1}
\end{array}\right] ; \\
\mathrm{A} & =\left[\begin{array}{cccccccc}
B 1 & g_{0} I & 0 & \ldots & 0 & 0 & 0 \\
g_{2} I & B 1 & g_{0} I & \ldots & 0 & 0 & 0 \\
g_{3} I & g_{2} I & B 1 & & 0 & 0 & 0 \\
\vdots & \vdots & \vdots & \ddots & g_{0} I & \vdots & \vdots \\
g_{N-3} I & g_{N-4} I & g_{N-5} I & \ddots & B 1 & g_{0} I & 0 \\
g_{N-2} I & g_{N-3} I & g_{N-4} I & \ldots & g_{2} I & B 1 & g_{0} I \\
g_{N-1} I & g_{N-2} I & g_{N-3} I & & g_{3} I & g_{2} I & B 1
\end{array}\right]
\end{aligned}
$$

Thecoefficients-matrix $A$ is of order $(\mathrm{N}-1)^{2} \times(\mathrm{N}-1)^{2}$, whereB1 is $\mathrm{N}-1 \times \mathrm{N}-1$ block matrix, $\mathrm{I}$ is $\mathrm{N}-1 \times \mathrm{N}-1$ identity matrix

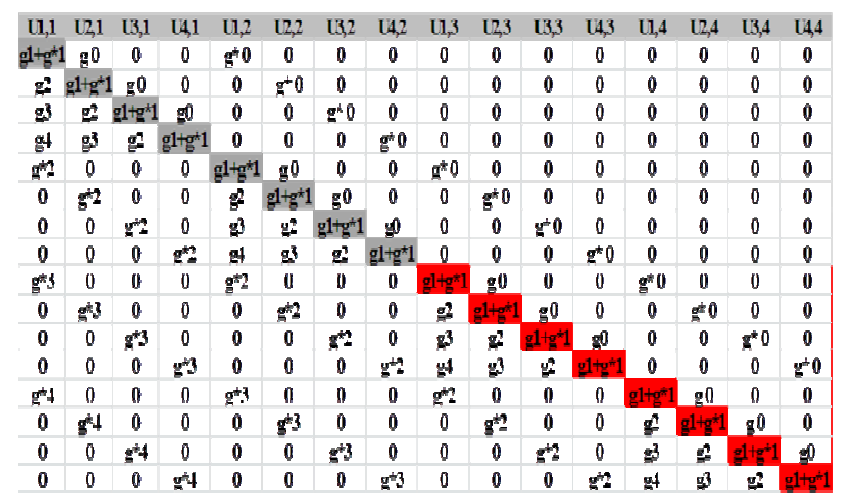

Figure5. Lexicographical ordering; formula (14), $N=5 ; 0<\alpha, \beta \leq 2$.

Theorem: the coefficients-Matrix for system (9) is nonsingular, so the system (12) has unique solution.

Proof: fromGershgorin's, theOremthe eigenvalues of $(\mathrm{N}$ $1)^{2} \times(\mathrm{N}-1)^{2}$, A matrix lie in union of $(\mathrm{N}-1)^{2}$ circles centered at $\mathrm{a}_{\mathrm{ii}}$ with radius $\mathrm{R}_{\mathrm{i}}=\sum_{j=1}^{N}\left|a_{i j}\right|$.

From figure 5 and equation (13), also $g_{k}^{\gamma}, \sum_{k=0}^{\infty} g_{k}^{\gamma}=$ $0 ; g_{k}^{\gamma}>0$ for $k \geq 2 ; \sum_{k=0}^{\infty} g_{k}^{\gamma}<0$; then

$$
\sum_{k=0}^{i+1} g_{k}^{\gamma}<-g_{1}^{\gamma}, \gamma=\alpha, \beta
$$

$$
\begin{aligned}
& a_{i i}=\left(g_{1}+g_{1}^{*}\right)=-\left(r_{1} \alpha+r_{2} \beta\right) \text {, then since } \\
& \qquad \mathrm{R}_{i}=\sum_{\substack{j=1 \\
j \neq i}}^{N}\left|a_{i j}\right|=\sum_{\substack{k=0 \\
k \neq i}}^{i+1}\left|a_{i k}\right|+\sum_{\substack{s=0 \\
s \neq i}}^{i+1}\left|a_{i s}\right| \\
& =\sum_{\substack{k=0 \\
k \neq i}}^{i+1} g_{k}+ \\
& <-\left(r_{1} \alpha+r_{2} \beta\right)=\left(g_{1}+g_{1}^{*}\right)
\end{aligned}
$$

Now we have that $\mathrm{a}_{\mathrm{ii}}+\mathrm{R}_{\mathrm{i}}<0, \mathrm{a}_{\mathrm{ii}}-\mathrm{R}_{\mathrm{i}}>-2\left(r_{1} \alpha+r_{2} \beta\right)$, this strict inequality true at ( $\alpha$ and $\beta$ ) fractional, then the eigenvalues of $\mathrm{A}$ are $\left(0>\lambda>-2\left(r_{1} \alpha+r_{2} \beta\right)\right)$, this mean that: $\mathrm{A}$ is invertible, and since $\mathrm{R}_{\mathrm{i}}<\left(g_{1}+g_{1}^{*}\right)=\mathrm{a}_{\mathrm{ii}}$, i.e $\mathrm{A}$ is strictly diagonal dominant, then $\mathrm{A}$ is non-singular and system (7) has a unique solution for $(1<\alpha, \beta<2)$. Moreover A is L-matrix,

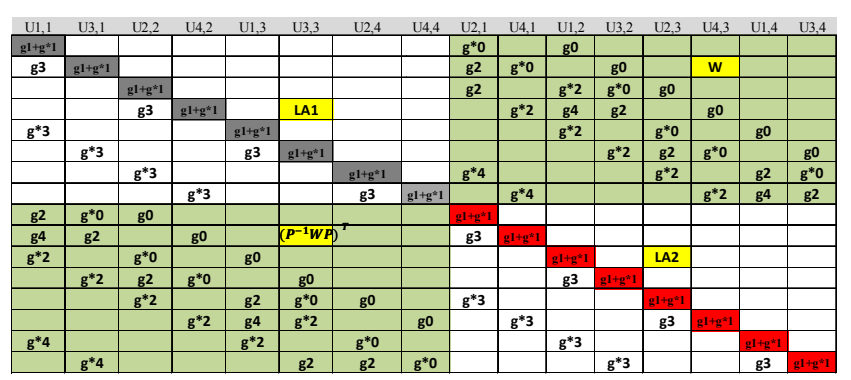

Figure6.Chequer-board ordering; formula (14), $N=5 ; 1<\alpha, \beta \leq 2$.

SOR and KSOR [1- 3, 8 - 11]:

Solving large linear systems is a problem in itself. Many authors considered the iterative techniques for solving such systems. The successive over relaxation (SOR) method $[1,2,8]$ is one of the most used iterative methods tosolve large systems. The SOR method depends on a relaxation parameter $\omega \in(0,2)$. The choice of $\omega$ (the optimum value $\left.\omega_{\text {opt }}\right)$ which minimizes the spectral radius of the iteration matrix $T_{S O R}$ is the most important and difficult part in the use of the SOR method. Although, there is a formula for determining $\omega_{\text {opt }}$ in case of system (5) there is no method for the selection of $\omega_{\text {opt }}$ in general. The KSOR introduced in [3] in 2012 is another variant of the SOR method in which the domain of the relaxation parameter is extended to R-[-2,0] instead of $(0,2)$.

$$
\begin{aligned}
& T_{S O R}=(D-\omega L)^{-1}((1-\omega) D+\omega U), \\
& \text { where } \omega \in(0,2) . \\
& T_{K S O R}=\left(\left(1+\omega^{*}\right) D-\omega^{*} L\right)^{-1}\left(D+\omega^{*} U\right), \\
& \text { where } \omega^{*} \in R-[-2,0] .
\end{aligned}
$$

The Precondition technique [8-11]: precondition techniques are used to accelerate the rate of convergence of iterative methods. In solving linear systems iteratively there are many varieties of preconditions. In general, a 
good preconditioned $\mathrm{P}$ should meet the following requirements:

- The preconditioned system should be easy to solve.

- The preconditioner should be cheap to construct andapply.

The first property means that the preconditioned iteration should converge rapidly, whilethe second ensures that each iteration is not too expensive. Notice that these two requirements are in competition with each other. It is necessary to strike a balance between the two needs with a good preconditioner. Sometimes the preconditioned matrix is well structured than the original matrix. Figure(6) corresponding to the chequer-board ordering (odd even ordering) the numbers $g_{4}^{*}$ appears in the element position $(7,9)$ and $(8,10)$ can be annihilated by using a precondition matrix without affecting the structure of the block sub matrices as illustrated in figure (7) and figure (8)

\begin{tabular}{|c|c|c|c|c|}
\hline$i-j$ & 8 & 9 & 10 & 11 \\
\hline 7 & 0 & $\mathrm{~g} * 4$ & 0 & 0 \\
\hline 8 & $g 1+g^{*} 1$ & 0 & $g * 4$ & 0 \\
\hline 9 & 0 & $\mathrm{~g} 1+\mathrm{g} * 1$ & 0 & 0 \\
\hline 10 & 0 & g3 & $g 1+g * 1$ & 0 \\
\hline
\end{tabular}

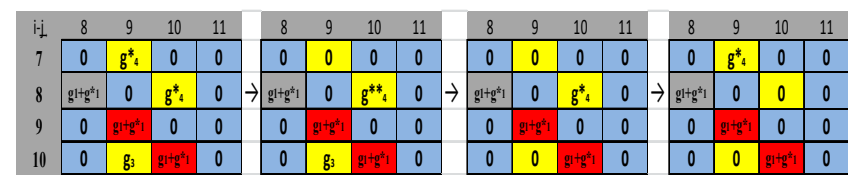

The preconditioned system is written in the form

$$
P(A U=f) \text { or } A_{p} U=f_{p}
$$

Where, $\mathrm{P}=\mathrm{P}_{2} \mathrm{P}_{1}$

With

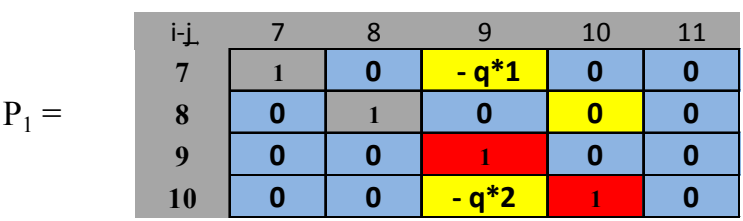

\begin{tabular}{|c|c|c|c|c|c|c|}
\hline \multirow{5}{*}{$\mathrm{P}_{2}=$} & \multirow{3}{*}{$\begin{array}{c}i-j \\
7\end{array}$} & 7 & 8 & 9 & 10 & 11 \\
\hline & & 1 & 0 & 0 & 0 & 0 \\
\hline & & 0 & 1 & 0 & $-q^{*} 1$ & 0 \\
\hline & 9 & 0 & 0 & 1 & 0 & 0 \\
\hline & 10 & 0 & 0 & 0 & 1 & 0 \\
\hline
\end{tabular}

For more details you can see [8] and [9].

In general, if we have a coefficient matrix of order $2 \ell$ with submatrices of order $\ell$ this precondition must be designed to annihilate the elements in positions $(\ell-1, \ell+$ 1 ) and the elements in positions $(\ell+2, \ell+1)$ and then annihilate the element in position $(\ell, \ell+2)$.

Numerical example: consider the structure of the coefficient matrix in figure (6) after calculating the numerical values of the coefficient matrix take the form illustrated in figure (7)

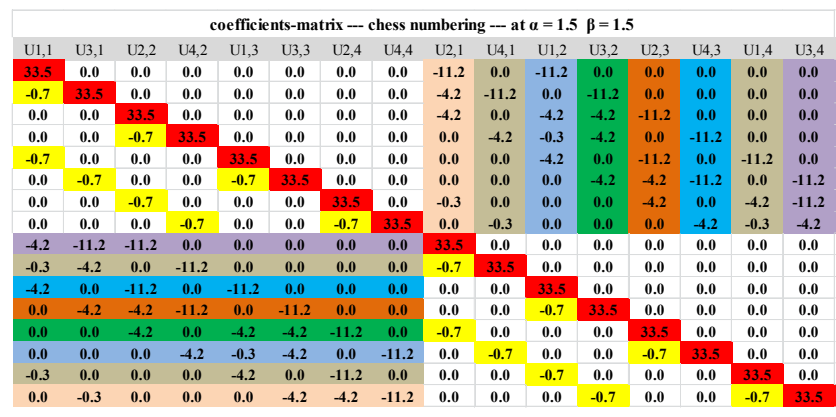

Figure7. Coefficient matrix for formula (14), $N=5 ; \alpha=\beta=1.5$.

As a result of applying the precondition described above we obtain the following matrix

coefficients-matrix; at $\alpha=\beta-1.5$ - Chess numbering, , after using Preconditions to change A(7,9) and A(8,10) to zero $\begin{array}{llllllllllllllll}0.000 & 0.000 & 0.000 & 0.000 & 0.000 & 0.000 & 0.000 & -0.333 & 0.000 & -0.333 & 0.000 & 0.000 & 0.000 & 0.000 & 0.000\end{array}$ $\begin{array}{llllllllllllllllll}-0.021 & 1.000 & 0.000 & 0.000 & 0.000 & 0.000 & 0.000 & 0.000 & -0.125 & -0.333 & 0.000 & -0.333 & 0.000 & 0.000 & 0.000 & 0.000\end{array}$

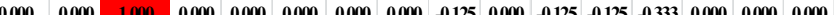
$\begin{array}{lllllllllllllllllll}0.000 & 0.000 & -0.021 & 1.000 & 0.000 & 0.000 & 0.000 & 0.000 & 0.000 & -0.125 & -0.008 & -0.125 & 0.000 & -0.333 & 0.000 & 0.000\end{array}$

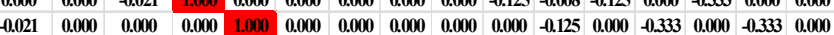

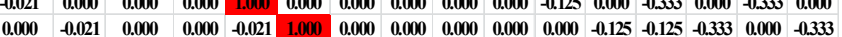
$\begin{array}{rrrrrrrrrrrrrrrrr}0.000 & -0.021 & 0.000 & 0.000 & -0.021 & 1.000 & 0.000 & 0.000 & 0.000 & 0.000 & 0.000 & -0.125 & -0.125 & -0.333 & 0.000 & -0.333 \\ -0.001 & -0.003 & -0.023 & 0.000 & 0.000 & 0.000 & 1.000 & 0.000 & 0.000 & 0.000 & 0.000 & 0.000 & -0.125 & 0.000 & -0.125 & -0.333\end{array}$

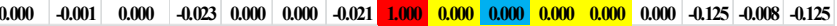
$\begin{array}{llllllllllllllll}-0.125 & -0.333 & -0.333 & 0.000 & 0.000 & 0.000 & 0.000 & 0.000 & 1.000 & 0.000 & 0.000 & 0.000 & 0.000 & 0.000 & 0.000 & 0.000\end{array}$ $\begin{array}{lllllllllllllllll}-0.010 & -0.132 & -0.007 & -0.333 & 0.000 & 0.000 & 0.000 & 0.000 & 0.000 & 1.000 & 0.000 & 0.000 & 0.000 & 0.000 & 0.000 & 0.000\end{array}$ $\begin{array}{llllllllllllllll}0.000 & 0.000 & -0.125 & 0.000 & -0.125 & -0.125 & -0.333 & 0.000 & -0.021 & 0.000 & 0.000 & 0.000 & 1.000 & 0.000 & 0.000 & 0.000\end{array}$ $\begin{array}{llllllllllllllll}0.000 & 0.000 & 0.000 & -0.125 & -0.008 & -0.125 & 0.000 & -0.333 & 0.000 & -0.021 & 0.000 & 0.000 & -0.021 & 1.000 & 0.000 & 0.000\end{array}$ $\begin{array}{llllllllllllllll}-0.008 & 0.000 & 0.000 & 0.000 & -0.125 & 0.000 & -0.333 & 0.000 & 0.000 & 0.000 & -0.021 & 0.000 & 0.000 & 0.000 & 1.000 & 0.000\end{array}$ $\begin{array}{llllllllllllllll}0.000 & -0.008 & 0.000 & 0.000 & 0.000 & -0.125 & -0.125 & -0.333 & 0.000 & 0.000 & 0.000 & -0.021 & 0.000 & 0.000 & -0.021 & 1.000\end{array}$

Figure8, the preconditioned matrix.

In the following table we summarize the results of calculating the spectral radius of the iteration matrices to both the SOR and the KSOR methods for the coefficient matrix given in figure (6) with different values of ( $\alpha$ and $\beta$ ), and for slandered coefficients-matrix (A) and preconditionmatrix (PA).

Table1.the values of the relaxation parameter $\omega$ and the corresponding spectral radius of the iteration matrix $\rho(T)$ for the SOR and the KSOR corresponding to the chess ordering.

\begin{tabular}{|c|c|c|c|c|c|c|c|c|c|}
\hline \multicolumn{2}{|c|}{ order } & \multicolumn{4}{|c|}{ SOR } & \multicolumn{4}{|c|}{ KSOR } \\
\hline$\alpha$ & $\beta$ & $\omega$ & $\rho\left(\mathrm{T}_{\mathrm{A}}\right)$ & $\omega$ & $\rho\left(\mathbf{T}_{\mathbf{P A}}\right)$ & $\omega^{*}$ & $\rho^{*}\left(\mathrm{~T}_{\mathrm{A}}\right)$ & $\omega^{*}$ & $\rho^{*}\left(\mathbf{T}_{\mathbf{P A}}\right)$ \\
\hline 1.2 & 1.2 & 1.05431 & 0.06955 & 1.05231 & 0.06135 & -19.455 & 0.069 & -20.131 & 0.061 \\
\hline 1.4 & 1.2 & 1.06831 & 0.09548 & 1.06491 & 0.08931 & -15.635 & 0.095 & -16.404 & 0.089 \\
\hline 1.6 & 1.2 & 1.08651 & 0.12453 & 1.08151 & 0.11466 & -12.565 & 0.124 & -13.285 & 0.115 \\
\hline 1.8 & 1.2 & 1.10651 & 0.17721 & 1.10991 & 0.17366 & -10.378 & 0.176 & -10.095 & 0.174 \\
\hline
\end{tabular}

Where $\rho\left(T_{A}\right)$ is the spectral radius of the iteration matrix corresponding to the original system and $\rho\left(T_{P A}\right)$ is the spectral radius of the iteration matrix corresponding to the preconditioned system 


\section{Conclusions}

The memory and hereditary properties of fractional derivativesdoes not appear in the finite difference representation (7) it is similar tothe integer case except for the right hand side term as illustrated in the matrix structure in figure (2) and figure(3). In the finite difference form (9) and (10) for the fractional order case the behavior of the approximation of the fractional derivatives appears in the matrix structure as illustrated in figure (4), figure (5) and figure(6). The precondition introduced meets the second requirement of precondition, it is easy to construct.

The SOR and the KSOR have the same behavior but with different values of the relaxation parameters for both the original system and the preconditioned system as shown in table 1 .

The structure of the resultant algebraic system will be our concern in a next subsequent work!

This paper shows the stander formula of coefficient matrices respect to two numbering methods,

Also, it shows that the system in formula(9) has exact and has unique solution for $(1<\alpha, \beta<2)$.

\section{References}

[1] D. M. Young, Iterative solution of large linear systems, Academic Press, New York (1970).

[2] D. M. Young, Iterative methods for solving Partial difference equation of Elliptic type, Ph. D. thesis, Department of mathematics, Harvard university Cambridge, mass, may 1, 1950.
[3] I. K,Youssef, on the successive overrelaxation method, Journal of math. and statistics 8 (2): 172-180,2012.

[4] V. D.Beibalaev ,Ruslan P. Meilanov, the Dirihlet problem for the fractional Poisson's equation with caputoDerivatives, afinite difference approximation and a numerical solution. Thermal scince:year, val,16, 2012.

[5] K. B. Oldham and J. Spanier,the Fractional Calculus, Academic Press, New York, NY, USA, 1974.

[6] Lijuan $\mathrm{Su}$ and Pei Cheng, A Weighted Average Finite Difference Method for the Fractional Convection-Diffusion Equation, Hindawi Publishing Corporation Advances in Mathematical Physics Volume 2013.

[7] N. G. Abrashina-Zhadaeva and I. A. Timoshchenko, FiniteDifference Schemes for a Diffusion Equation with Fractional Derivatives in a Multidimensional Domain, ISSN 00122661, Vol. 49, Differential Equations, 2013.

[8] YousefSaad, Iterative Methods for Sparse Linear Systems, by the Society for Industrial and Applied Mathematics, 2003.

[9] D. J. Evans, M. M. Martins, M. E. Trigo, On preconditioned MSOR Iterations, Intern, J. Computer Math, Vol. 59, pp. 251-257.

[10] Michele Benzi, Preconditioning Techniques for Large Linear Systems, Journal of Computational Physics 182, 418-477 (2002).

[11] O. Settle, C. C. Douglas, I. Kim, D. Sheen, On the derivation of highest-order compact finitedifference schemes for the one- and two-dimensional poisson equation with dirichlet boundary conditions, SIAM Journal on Numerical Analysis, Vol. 51, No. 4, (2013). 